\title{
Author Correction: Selenium-GPX4 axis protects follicular helper T cells from ferroptosis
}

Yin Yao (D), Zhian Chen, Hao Zhang, Cailing Chen, Ming Zeng, Joseph Yunis (D), Yunbo Wei, Yanmin Wan, Naiqi Wang (D), Mingzhe Zhou, Chao Qiu, Qunxiong Zeng, Hong Sheng Ong, Hao Wang, Fadzai Victor Makota, Yang Yang, Zhaohui Yang, Nan Wang, Jun Deng, Chao Shen, Yan Xia, Lin Yuan, Zhaoqin Lian, Yike Deng, Cuilian Guo, Ao Huang, Pengcheng Zhou(D), Haibo Shi, Weitian Zhang, Hongliang Yi, Dongmei Li, Ming Xia, Jing Fu, Ning Wu, Judy B. de Haan, Nan Shen (1D,

Wenhong Zhang, Zheng Liu(D) and Di Yu(D)

Correction to: Nature Immunology https://doi.org/10.1038/s41590-021-00996-0, published online 19 August 2021

In the originally published version of this Article, there was an error in Extended Data Fig. 10c. Specifically, in Extended Data Fig. 10c, third panel to the right, the $y$-axis label (In CD4 ${ }^{+}$cells (\%)) was incorrect. The correct label has been amended to read: "In $\mathrm{CD} 4{ }^{+} \mathrm{CXCR}^{+}$ cells (\%)."

The change has been made to the online version of the Article.

Published online: 4 October 2021

https://doi.org/10.1038/s41590-021-01063-4

(c) The Author(s), under exclusive licence to Springer Nature America, Inc. 2021 